\title{
Isolated lateral rectus myositis in a nineteen-month-old male patient - case report
}

\author{
Klaudia Rakusiewicz, Krystyna Kanigowska, Wojciech Hautz, Agnieszka Czeszyk, Małgorzata Danowska, \\ Joanna Jędrzejczak-Młodziejewska, Anna Rogowska, Marta Wyszyńska
}

Department of Pediatric Ophthalmology, Children's Memorial Health Institute, Warsaw, Poland

\begin{abstract}
Aim of the study: To present a case of lateral rectus myositis in a 19-month-old male patient.

Case report: The 19-month-old male patient was admitted to the clinic because of exophthalmos of the right eye for diagnosis and further treatment. Based on the clinical manifestation, and after other potential causes had been ruled out, the patient was diagnosed with ocular myositis. Treatment with steroids and antibiotics resulted in resolution of symptoms.
\end{abstract}

Conclusions: Ocular myositis is an isolated inflammation of the extraocular muscles, manifested by swollen eyelids, exophthalmos, pain upon eye movement and diplopia. Ocular myositis is an extremely rare disease which occurs in pediatric populations. To the best of our knowledge, this is the first documented case of myositis in a very young child.

KEY WORDS: ocular myositis, lateral rectus, extraocular muscles, exophthalmos, children.

\section{INTRODUCTION}

Ocular myositis (extraocular muscle inflammation) is a non-specific, idiopathic and non-infectious inflammatory condition which affects one or several extraocular muscles [1-3]. It usually affects young patients in the second to third decade of life and is more prevalent among females $[1,2,4,5]$. The clinical features of ocular myositis include: eyelid edema, conjunctival injection, pain exacerbating during eye movements, diplopia and ptosis [1-4]. A number of other medical conditions occurring in the orbital region, such as inflammation, neoplasms and vascular disorders, may mimic ocular myositis [3]. The treatment of choice for ocular myositis is anti-inflammatory therapy with non-steroid anti-inflammatory drugs and steroids $[2,3]$. The goals of therapy include improvement of the patient's comfort level, reduction of disease duration and relapse prevention [2]. In most cases, the therapy results in quick improvement and disease remission within several days or weeks [3].

\section{CASE REPORT}

The nineteen-month-old male patient was admitted to the Ophthalmology Clinic at the Children's Memorial Health Institute due to exophthalmos of the right eye with onset two weeks before. Due to conjunctivitis, diagnosed in another center, he was undergoing topical antibiotic therapy at that time. The patient had no concomitant systemic diseases or symptoms of allergy. During ophthalmological examination, he was diagnosed with right eye exophthalmos with preserved normal motility of both eyes, except for a minor limitation of right eye abduction. Clinical assessment confirmed a minor edema of the superior eyelid without increased skin warmth, redness or other symptoms of inflammation (Figure 1). Biomicroscopy did not identify changes in the anterior segments of either eye and both fundi were within normal limits. Ocular ultrasound showed a thickened lateral rectus muscle of the right eye (Figure 2). Cranial and orbital magnetic resonance imaging identified an abnormal mass $(16 \times 21 \times 33 \mathrm{~mm})$ adjacent to the lateral wall of the right orbit and attached to the lateral rectus (Figure 3). However, the imaging examination did not reveal any inflammatory changes in the adjoining structures. Due to a suspected neoplastic lesion, surgical

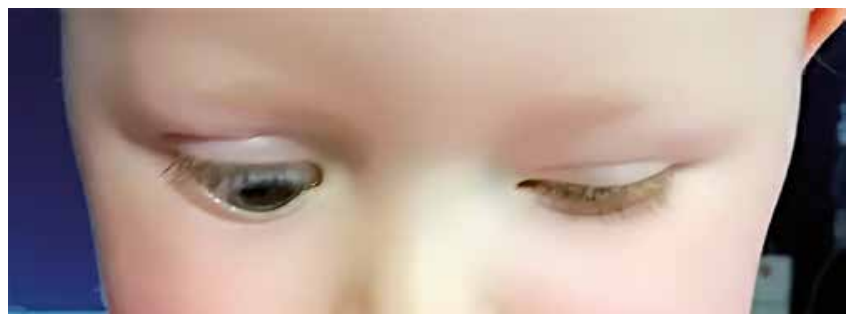

Figure 1. Exophthalmos of the right eye in the nineteen-month-old male patient 


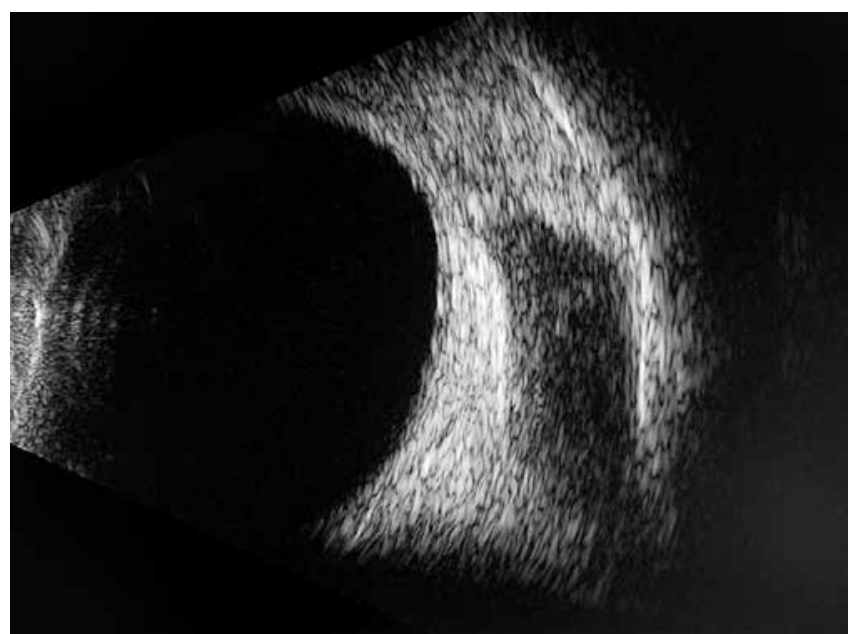

Figure 2. Ultrasound image - a thickened extraocular lateral rectus muscle

biopsy was recommended, following an oncological consultation, in order to establish a final diagnosis. In order to rule out potential metastases to other organs, the diagnostic process was extended by including chest computed tomography and abdominal/mediastinal ultrasound. The imaging examinations did not reveal any lesions with features of focal metastatic areas.

A basic set of laboratory tests (complete blood cell count with differential count, electrolyte panel, blood glucose level) as well as inflammatory marker tests (erythrocyte sedimentation rate - ESR and C-reactive protein - CRP) produced results within normal limits. A number of blood tests were ordered to verify normal renal, hepatic and thyroid functions, including the levels of uric acid, lactate dehydrogenase $(\mathrm{LDH})$, aspartate transaminase (AspAT), alanine transaminase (AlAT), creatinine, urea, glomerular filtration rate (GFR), thyroid stimulating hormone (TSH), Ft3, Ft4 and anti-TPO, but none of these tests showed abnormal results. The patient was also consulted by a laryngologist, who ex-

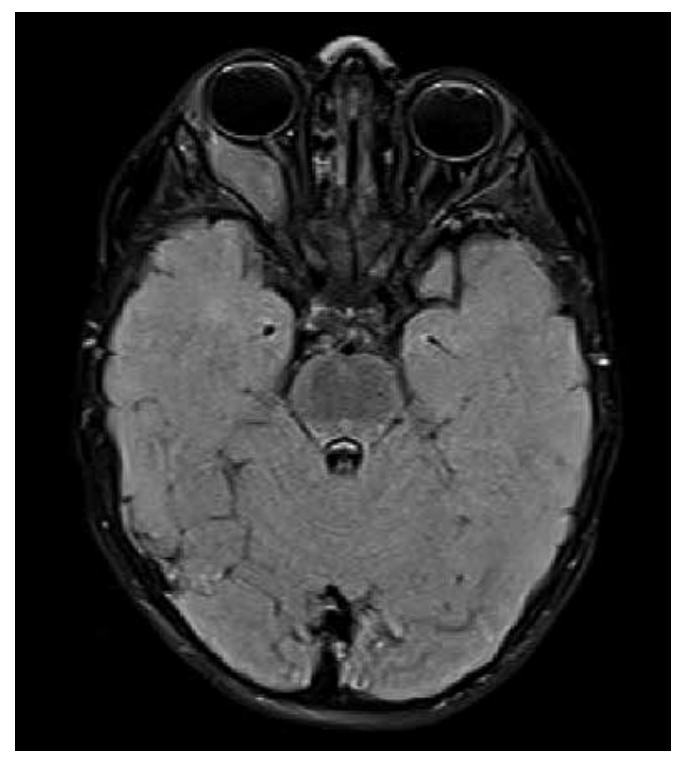

Figure 3. Abnormal mass in magnetic resonance imaging cluded lesions located in the sinuses which might have potentially resulted in upper eyelid edema and exophthalmos. Ultrasound guided right orbit biopsy was performed under general anesthesia. Histopathological tests identified the presence of adipose tissue containing macrophages and eosinophils but without neoplastic cells. As it was suspected that the biopsy specimen might have been acquired from the edge of the lesion proper, following oncological, neurosurgical and pathomorphological consults, it was decided to repeat the surgical biopsy. The new specimen consisted of fibrotic connective tissue and adipose tissue and all the structures within the specimen contained inflammatory infiltration cells. However, no neoplastic cells were identified in the sample material. As the diagnosis remained ambiguous, systemic therapy was not initiated at this stage. The subsequent imaging examination indicated a spontaneous reduction of the mass, which exposed a visibly thickened lateral rectus of the right eye. As an inflammatory process was suspected, after potential tumor metastasis as well as other systemic and localized diseases had been excluded, it was decided to initiate antiinflammatory steroid therapy and antibiotic therapy. Exophthalmos resolved after one week of therapy. The dynamics and characteristics of the clinical manifestation in this case confirmed the diagnosis of isolated ocular myositis. Followup magnetic resonance imaging performed six months later indicated a significant reduction of the muscle's thickness. The asymmetry between the thicknesses of lateral rectus muscles in both eyes was minor and probably due to post-inflammatory fibrosis (Figure 4). Based on the clinical manifestation described above, the patient was finally diagnosed with isolated lateral rectus inflammation in the right eye. The patient has been monitored for two years and has not relapsed to date.

\section{DISCUSSION}

The causes of unilateral proptosis are diverse among pediatric and adult patients. The most common causes of

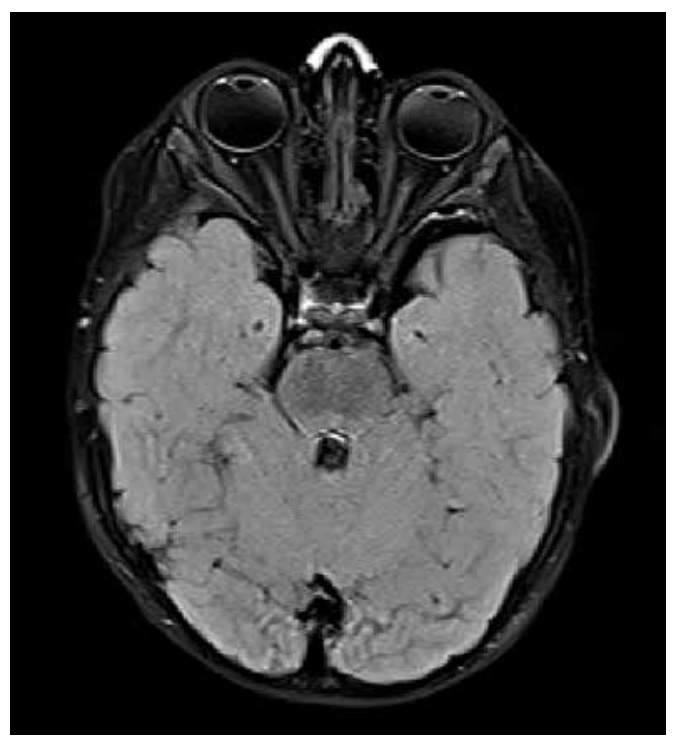

Figure 4. Magnetic resonance imaging result six months after diagnosis of exophthalmos 
exophthalmos in children are neoplastic lesions and orbital cellulitis $[6,7]$.

The term orbital cellulitis is used to describe orbital tissue inflammation with infectious etiology. The disease is associated with erythema, eyelid edema, pain and purulent discharge, usually concomitant with fever $[5,8]$. The most common sources of infection are inflammatory processes located in the sinuses, the eyelids and the skin of the adjacent tissues [8]. Based on a study including 122 pediatric patients, Santos et al. [9] observed that $40.2 \%$ of ocular cellulitis cases were caused by inflammation located in the sinuses. The patient described here did not exhibit features characteristic of inflammation such as redness, increased body temperature or abnormal ESR and CRP levels. Fever and airway infection were also excluded. Laryngological examination did not identify abnormalities in the paranasal sinuses, which was later confirmed by magnetic resonance imaging. Therefore, the initial differential diagnosis did not include changes with inflammatory etiology.

Considering the patient's age and the most prevalent causes of exophthalmos, the tentative diagnosis in this case was a neoplastic disease. Proliferative disorders located in the orbit may be benign, malignant or constitute focal areas of metastasis.

The most common benign tumor located within the orbit is capillary hemangioma, within which endothelial cell hyperplasia with narrow vascular channels is found [10-13]. In most cases, the capillary hemangiomas are located on the upper eyelid, so that the skin of the eyelids is raised and reddened. Less often the capillary hemangiomas are located deep in the orbit; then the skin is normal $[12,13]$. It is characteristic that the lesions appear a few weeks after birth and are initially small and then grow rapidly within a few months [11]. Capillary hemangiomas may increase when the baby cries due to blood stasis and increased pressure [11]. However, in our patient we did not find any changes on the eyelid skin or increase in the size of the exophthalmos over time. Exophthalmos is also a typical symptom for metastatic foci located in the orbit. In children the most common metastatic tumor is neuroblastoma, originally located in the adrenal, retroperitoneal or mediastinal space [14]. The most prevalent malignant tumor affecting this region in children is rhabdomyosarcoma (RMS) [15]. 70\% of RMS cases manifest in the first decade of life, usually affecting boys rather than girls $[15,16]$. In $80-100 \%$ of cases, exophthalmos develops quickly, usually within several days $[15,16]$. It is recommended that the diagnostic process include imaging of the cranial and orbital regions. If the characteristic radiological image is confirmed, further management should include surgical biopsy in order to confirm the diagnosis based on a histopathological specimen $[15,16]$. In the case described here, magnetic resonance imaging identified an irregular mass proximal to the lateral wall of the right orbit, which suggested a neoplastic lesion. Based on the current recommendations, the patient underwent a surgical biopsy twice with both results being negative for neoplastic cells.
Apart from the most prevalent diseases, differential diagnosis of exophthalmos should also include disorders such as acute dacryoadenitis, thyroid orbitopathy, idiopathic orbital inflammation, sarcoidosis and Tolosa-Hunt syndrome [2, 3].

Thyroid orbitopathy is a self-limiting autoimmune process localized in the orbit and in eighty percent of cases it is associated with hyperthyroidism [17]. The disease affects females six times more often than males, usually in the third and fourth decades of life [17]. A typical feature of thyroid orbitopathy is bilateral exophthalmos and only $5 \%$ of cases exhibit unilateral proptosis. Thyroid orbitopathy is associated with edema, fibrosis, fatty infiltration and inflammation affecting the extraocular muscles. In order to rule out thyroid orbitopathy as the underlying cause of exophthalmos, the patient underwent laboratory tests to determine the levels of thyroid hormone and thyroid-specific antibodies as well as mediastinum ultrasound imaging, with all the results being within normal limits.

Due to evident thickening of the extraocular muscle visible in magnetic resonance imaging, following the elimination of other probable disease processes and considering the overall clinical manifestation, the tentative diagnosis was ocular myositis. The disease is a subtype of idiopathic orbital inflammation (IOI), which used to be referred to as orbital pseudotumor. IOI is a non-infectious, non-neoplastic inflammation of the orbital tissues $[5,18]$ while ocular myositis selectively affects one or more extraocular muscles $[3,19]$. It affects females more often than males $[3,19]$. Based on proprietary analysis, Schoser et al. [3] concluded that the incidence of ocular myositis is the highest in the second and third decades of life. Yan et al. [19] analyzed 44 cases of ocular myositis and the average patient's age at diagnosis was 39.1 years. According to the authors' best knowledge, ocular myositis has not been described to date in such a young patient. In order to diagnose ocular myositis, it is recommended to perform cranial and orbital MRI, which may indicate thickening of the inflamed muscle $[3,20]$. Biopsy is usually not indicated in order to confirm the diagnosis [3]. In the available literature, the authors rarely describe muscle biopsy with histopathological results indicating the presence of plasma cells, lymphocytes and macrophages [3]. Ocular myositis is associated with increased levels of creatine kinase (CK) and $\mathrm{LDH}[1]$. In this case, the $\mathrm{LDH}$ level remained normal but the CK level was not tested. Due to the inflammatory etiology, CRP and ESR are usually elevated but these markers are not specific to this particular disease [5]. A positive response to corticosteroid therapy is helpful in defining the final diagnosis $[2,3,20]$. In the discussed case, the levels of inflammatory markers remained normal. Following the implementation of anti-inflammatory therapy, the clinical condition of the patient improved significantly and the thickening of the muscle remarkably decreased, as confirmed by imaging examinations. Yan et al. [19] analyzed the clinical data of 44 patients diagnosed with ocular myositis and described disease relapse in $81.8 \%$ of cases. The nineteen-month-old patient described above has been monitored for the last two years and has not relapsed to date. 


\section{CONCLUSIONS}

Isolated ocular myositis is a rare disease in children. However, its significance in differential diagnosis of orbital lesions associated with exophthalmos should not be underestimated.
In the available literature, a case of ocular myositis has not been described to date in such a young patient.

\section{DISCLOSURE}

The authors declare no conflict of interest.

\section{References}

1. Aquilina N, Bugeja V, Zahra C. A case of orbital myositis presenting with dizziness. Open Access Maced J Med Sci 2018; 6: 1278-1281.

2. Costa MR, Dumitrascu, OM, Gordon LK. Orbital myositis: diagnosis and menagement. Curr Allergy Asthma Rep 2009; 9: 316-323.

3. Schoser BG. Ocular myositis: diagnostic assessment, differential diagnoses, and therapy of a rare muscle disease - five new cases and review. Clin Ophthalmol 2007; 1:37-42.

4. Scott IU, Siatkowski RM. Idiopathic orbital myositis. Curr Opin Rheumatol 1997; 9: 504-512.

5. Fraser CL, Skalicky SE, Gurbaxani A, McCluskey P. Ocular myositis. Curr Allergy Asthma Rep 2013; 13: 315-321.

6. Sethi A, Ghose S, Gujral S, et al. Childhood proptosis: The invaluable, though often overlooked peripheral blood smear. Indian J Ophthalmol 2001; 49: 121-123.

7. Altonbary Y, Mansour AK, Sarhan M, et al. Proptosis is a pediatric dilemma. Ann Pediatr Child Health 2015; 3.

8. Tsirouki T, Dastiridou Al, Ibánez Flores N, et al. Orbital cellulitis. Surv Ophthalmol 2018; 63:534-553.

9. Santos JC, Pinto S, Ferreira S, et al. Pediatric preseptal and orbital cellulitis: A 10-year experience. Int J Pediatr Otorhinolaryngol 2019; 120: 82-88.

10. Bang GM, Setabutr P. Periocular capillary hemangiomas: indications and options for treatment. Middle East Afr J Ophthalmol 2010; 17: 121-128.

11. Haggstrom AN, Drolet BA, Baselga $E$, et al. Prospective study of infantile hemangiomas: clinical characteristics predicting complications and treatment. Pediatrics 2006; 118: 882-887.

12. Kushner BJ. Hemangiomas. Arch Oftalmol 2000; 118: 835-836.

13. Ni N, Wagner RS, Langer P, Guo S. New developments in the management of periocular capillary hemangioma in children. J Pediatr Ophthalmol Strabismus 2011; 48: 269-277.

14. Harreld JH, Bratton EM, Federico SM, et al. Orbital metastasis is associated with decreased survival in stage M neuroblastoma. Pediatr Blood Cancer 2016; 63: 627-633.

15. Rao AA, Naheedy JH, Chen JY, et al. A clinical update and radiologic review of pediatric orbital and ocular tumors. J Oncol 2013; 2013: 975908.

16. Jurdy L, Merks JH, Pieters BR, et al. Orbital rhabdomyosarcomas: A review. Saudi J Ophthalmol 2013; 27: 167-175.

17. Maheshwari R, Weis E. Thyroid associated orbitopathy. Indian J Ophthalmol 2012; 60: 87-93.

18. Yuen SJ, Rubin PA. Idiopathic orbital inflammation: distribution, clinical features, and treatment outcome. Arch Ophthalmol 2003; 121: 491-499.

19. Yan J, Wu P. Idiopathic orbital myositis. J Craniofac Surg 2014; 25: 884-887.

20. Muralidhar R, Gautam K, Christopher D, et al. Isolated superior oblique myositis causing acquired Brown's syndrome. Indian J Ophthalmol 2015; 63:340-341. 